\title{
LA-UR-19-27364
}

Approved for public release; distribution is unlimited.

Title: $\quad$ Modeling Political Instability in Pakistan

Author(s): $\quad$ Goodloe, Oscar Laurence

Fairchild, Geoffrey

Intended for: Keepin Summer School end-of-program student presentations.

Issued: 
Disclaimer:

Los Alamos National Laboratory, an affirmative action/equal opportunity employer, is operated by Triad National Security, LLC for the National Nuclear Security Administration of U.S. Department of Energy under contract 89233218CNA000001. By approving this article, the publisher recognizes that the U.S. Government retains nonexclusive, royalty-free license to publish or reproduce the published form of this contribution, or to allow others to do so, for U.S. Government purposes. Los Alamos National Laboratory requests that the publisher identify this article as work performed under the auspices of the U.S. Department of Energy. Los Alamos National Laboratory strongly supports academic freedom and a researcher's right to publish; as an institution, however, the Laboratory does not endorse the viewpoint of a publication or guarantee its technical correctness. 


\section{Modeling Political Instability in Pakistan}

\section{Oscar Goodloe $^{1,2}$, Geoffrey Fairchild ${ }^{1}$}

- Los Alamos NATIONAL LABORATORY EST.1943
${ }^{1}$ Los Alamos National Laboratory Analytics, Intelligence, and Technology (A) Information Systems and Modeling (A-1)

2Arizona State University 


\section{Summer Fun (Meow Wolf)}
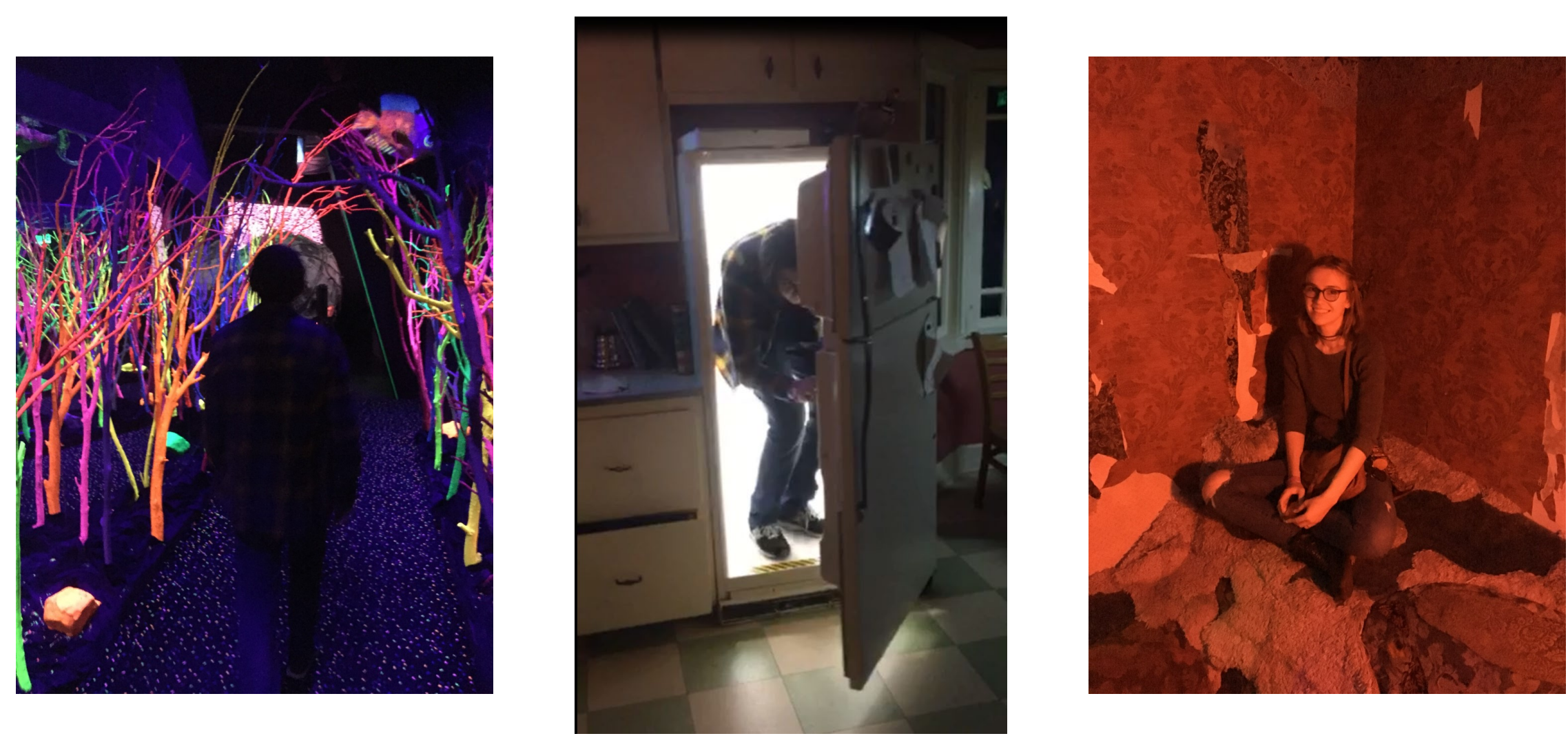


\section{Oscar Goodloe (A-1)}

- Educational Background

o B.S. Applied Mathematics. ASU, 2019

- A (Analytics, Intelligence, and Technology)

○ A-1 (Information Systems and Modeling)

o Mentor: Geoffrey Fairchild

- Research
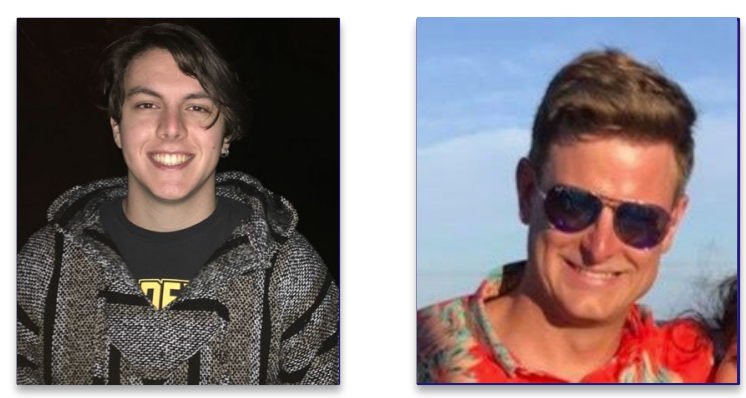

o At LANL: Political instability modeling.

o Elsewhere: Mathematical biology, network science, machine learning. 


\section{The PakMAN Project}

Goal: develop regional political instability proof-of-principle approach that can...

1. Assimilate heterogeneous data streams.

2. Quantify the contribution of different instability drivers.

3. Provide near real-time political instability forecasts with quantified uncertainty.

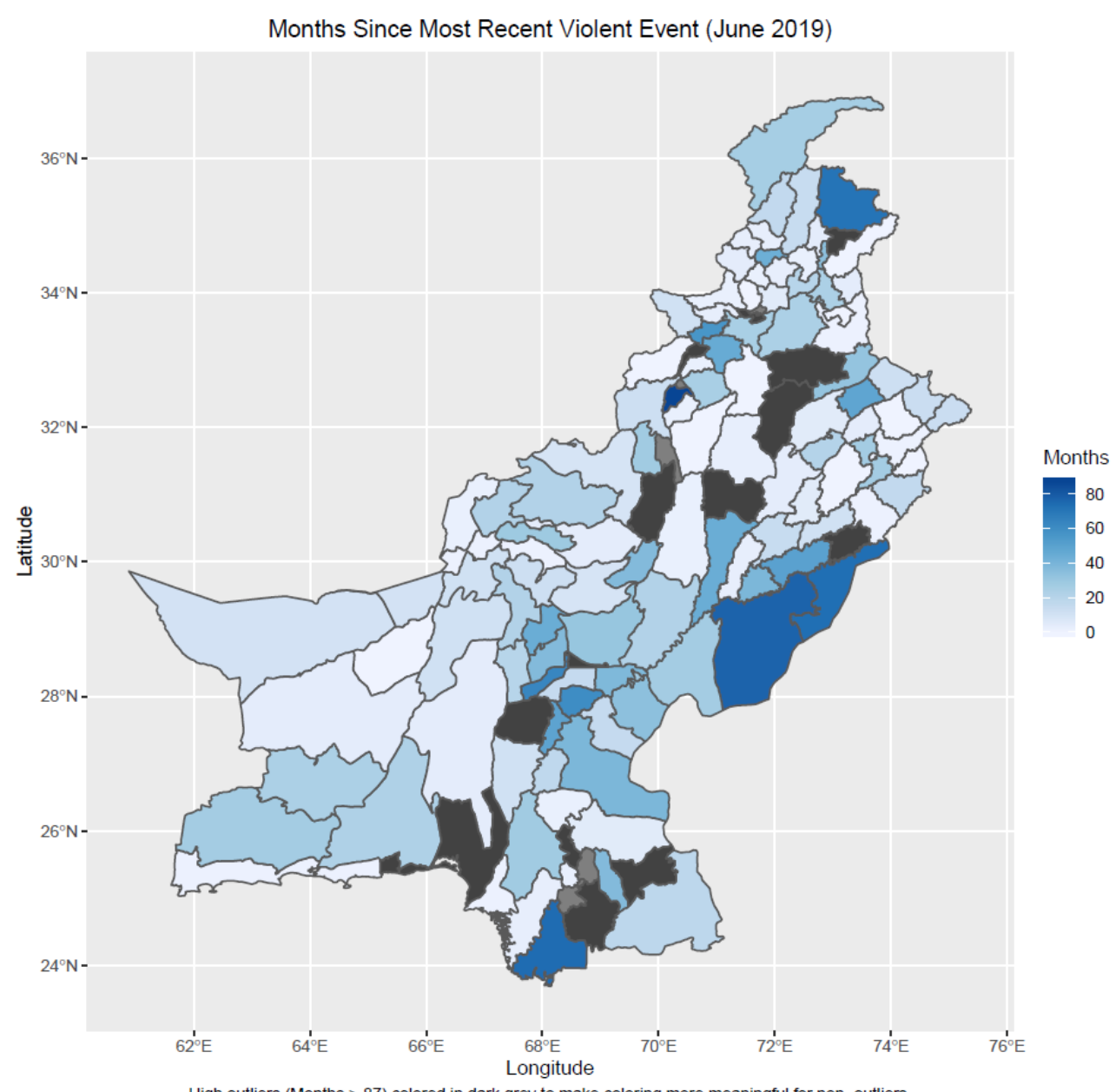




\section{Research Approach}

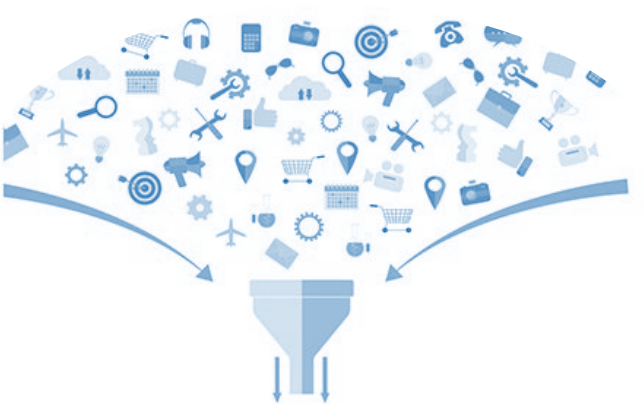

1) Identify, gather, and assimilate heterogeneous data streams.
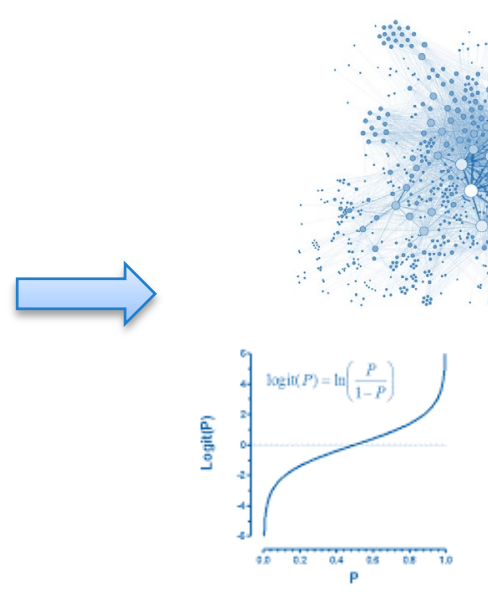

…․

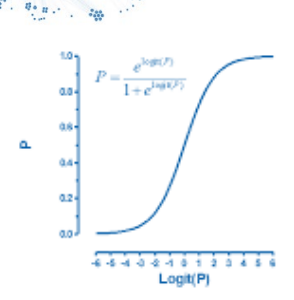

2) Develop and apply statistical, computational, and mathematical models.

\section{Notable Challenges:}

- Process has an incredibly complex state space (lurking included/excluded variable bias, strong correlations between predictors).

- Good candidates for outcome/response variables are hard to identify.

- Developing robust, largely automated pipelines for data collection and aggregation (continuity).

- Variation in spatial/temporal resolution between data sets.

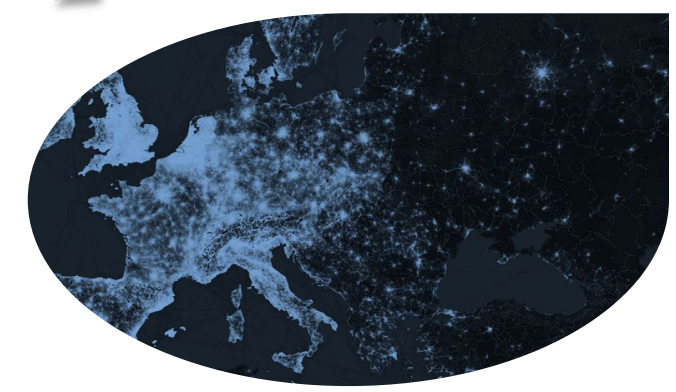

3) Produce real-time forecasts with quantified uncertainty. 


\section{Summary of Results (Preliminary Random Forest)}

- Outcome: sign of average event intensity at the district-month level $(-1,0,1)$

- Predictors: terrorism, violence history, leadership changes, forest coverage, climate

- Training: 25,000 observations, 70/30 train/test split, 5-fold CV.

Confusion Matrix for Random Forest Model

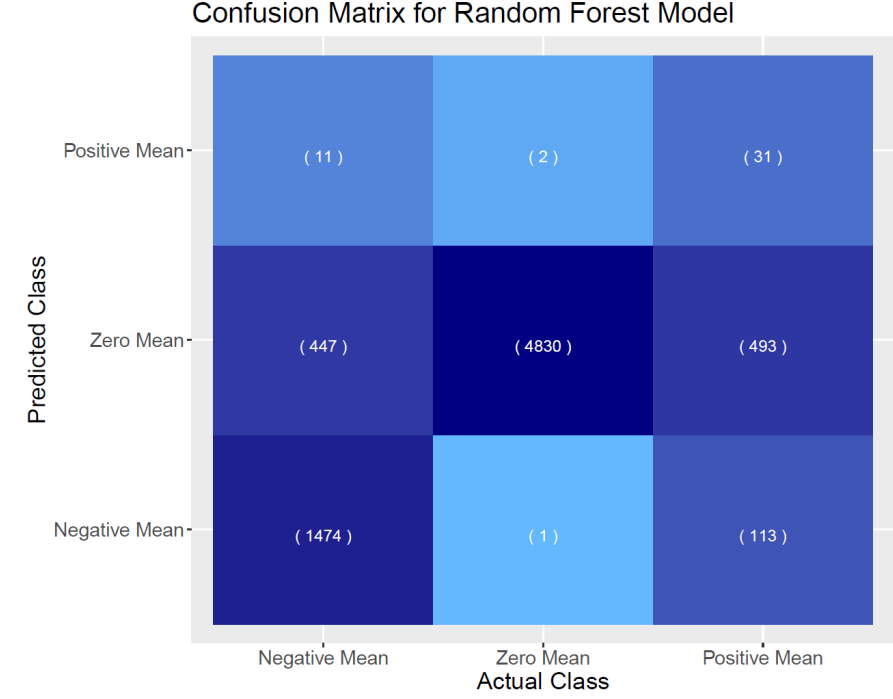

Overa71 statistics

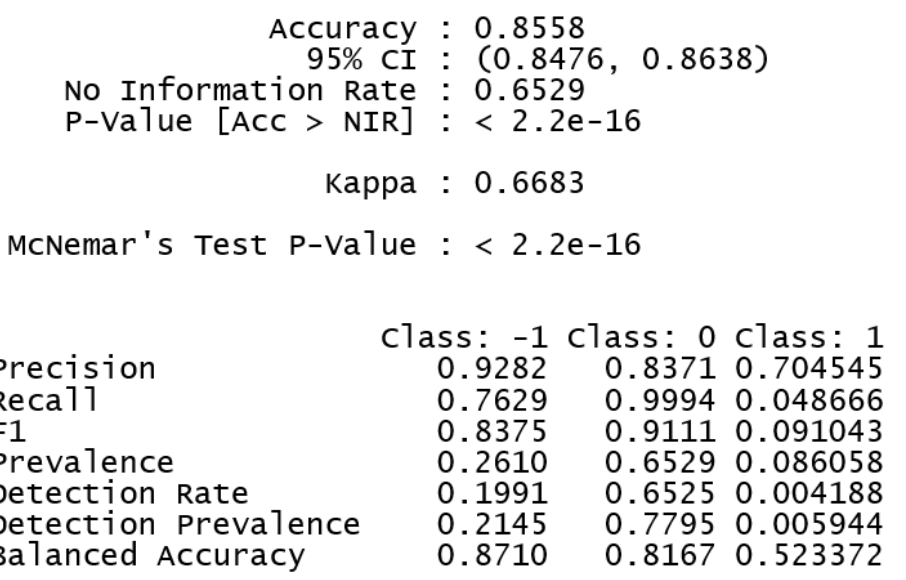

Variable importance

Months. Since. Violent. Event

Drought. Severity. Index

12.467402

wind. speed

12.244747

Max.Temperature

water.Deficit

12.052946

12.026732

11.915248

11.251656

Taliban.Events

Percent. Forest. Coverage

7. 166835

5.298586

charged. Events

0.000000 